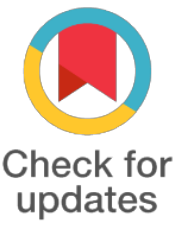

*For correspondence:

pavel_3@hotmail.com

Competing interests: The authors declare that no competing interests exist.

Received: 2017-06-14 Accepted: 2017-08-11 Published: 2017-09-05

Copyright The Author(s) 2017. This article is published with open access by BioMedPress (BMP).

This article is distributed under the terms of the Creative Commons Attribution License (CC-BY 4.0) which permits any use, distribution, and reproduction in any medium, provided the original author(s) and the source are credited.

\section{PLASMA LEVELS OF MMPS AND TIMP-1 IN BLADDER CANCER PATIENTS}

\author{
Pavel Yakovlev ${ }^{1}$, Ishchuk Tetiana ${ }^{2}$, Glavachek Diana², Savchuk Olexsiy ${ }^{2}$, \\ Falaleeva Tetiana², Ostapchenko Ludmila² \\ 1. Tarasa Shevchenko Blvd, 13 Kiev 01601 Ukraine \\ 2. Taras Shevchenko National University of Kyiv, ESC "The Institute of Biology", Ukraine.
}

\begin{abstract}
Background: Urinary bladder cancer (UBC) is a common disease worldwide with high mortality rate [1]. UBC is the ninth most frequently diagnosed cancer worldwide, with highest incidence rates observed in developed countries. About $75 \%$ of bladder cancer patients are men [2]. Matrix metalloproteinases (MMPs) and tissue inhibitors of metalloproteinases (TIMPs) are diagnostic tools in oncology, liver diseases, and rheumatoid arthritis, which may prove to be a valuable prognostic tool in clinical setting [3]. The aim of the present study was to assess the prognostic power of plasma MMPs and TIMP-1 in patients with UBC of different stages.
\end{abstract}

Methods: This study enrolled 29 patients with UBC (27 males, 2 females), aged 52-76 years. Preoperative study for all patients included blood test, blood chemistry, urine analysis, immunogram, computer tomography, tumor biopsy. The spread of cancer was characterized based on TNM clinical classification 7 th review (2009). Stage distribution of patients were as following: Stage I - 6 patients; Stage II - 5; Stage III - 7; Stage IV - 8 patients). The control group consisted of 15 healthy individuals ( 8 females and 22 males; aged $30-35$ years). Plasma levels of MMP-1, MMP-2, MMP-3, MMP-8, MMP-9 and TIMP-1 were determined by ELISA before the curative procedure and correlated afterwards with clinical parameters of the patient. Data analysis was conducted using Microsoft Excel 2010.

Results: Plasma levels of MMP-9 were significantly higher (by 1.5-2.0 times) in all patients with UBC compared to controls. Plasma level of MMP-8 in Stage III UBC patients was 1.2 times higher than in control group. The levels of MMP-1 and MMP-2 were increased compared to controls, and their elevation correlated with increase in cancer stage. Plasma level of MMP-3 in patients with bladder cancer Stage I, II and III were higher compared to controls by 1.3, 1.2 and 1.2 times respectively. High plasma levels of TIMP-1 were determined in patients with Stages III and IV, which raises question as to its role and function in advanced stage disease, as it may have a protective role in the mechanism of invasion by inhibiting extracellular matrix degradation.

Conclusion: In patients with urinary bladder cancer the plasma levels of MMP-1, MMP-2, MMP3, MMP-8, MMP-9 and TIMP-1 are elevated. We demonstrated that elevation pattern of MMP-1, MMP-2 and MMP-9 could serve as potential plasma marker for monitoring the cancer progression. 


\section{Keywords}

MMPs, TIMP-1, bladder cance

Funding

\section{References}

1. Ploeg M., Aben K.K., Kiemeney L.A. (2009). The present and future burden of urinary bladder cancer in the world. World J Urol, 27 (3), 289-293.

2. Cristiane Murta-Nascimento, Bernd J. Schmitz-Dräger, Maurice P. Zeegers, Gunnar Steineck Manolis Kogevinas, Francisco X. Real, Núria Malats. (2007). Epidemiology of urinary bladder cancer: from tumor development to patient's death. World J Urol ,25, 285-295.

3. Giannelli G, Erriquez R, lannone F, Marinosci F, Lapadula G, Antonaci S. (2004). MMP-2, MMP9, TIMP-1 and TIMP-2 levels in patients with rheumatoid arthritis and psoriatic arthritis. Clin Exp Rheumatol, 22(3), 335-3 38. 